\title{
A NEW TOOL FOR LARGE SCALE POWER SYSTEM TRANSIENT SECURITY ASSESSMENT
}

\author{
M. BOUDOUR ${ }^{1}$, A. HELLAL ${ }^{2}$ \\ ${ }^{1}$ Electrical Engineering Department, University of Sciences \& Technology, BP.32 El Alia, Algiers, Algeria \\ e-mail : mboudour@yahoo.com \\ ${ }^{2}$ Electrical Engineering Department, Polytechnic Institute, Algiers, Algeria, e-mail: aHellal@yahoo.fr
}

\begin{abstract}
In this paper, the nonlinear mapping relation between the transient energy margin and the generator power at different fault clearing time and load levels was established by using the self-organizing feature map. Lyapunov's direct method was used as a fast method to obtain the training set of the neural network. The transient security power limits of the generator at different fault clearing time and load levels were estimated very fast by the self-organizing mapping. A comparative study with the analytical sensitivity approach shows that the proposed method avoids the necessity of finding the analytical sensitivity of the transient energy margin to parameter changes, and can fast estimate transient security power limits at different fault clearing time and load levels of the system. Preliminary results carried out on a 7 machine-CIGRE test system are reported.
\end{abstract}

\section{Keywords}

Transient security limits, self-organizing map, Lyapunov energy function, Transient security assessment.

\section{Introduction}

The increasing necessity of operating power systems near to their limits because of delays in building new transmission facilities and power plants and also because of the new regulation systems are posing more and more interest in security assessment [1-4]. The transient stability power limits (power generation and transmission on important lies or load supply capabilities) are mainly related to the following factors: 1) the construction parameters of the network and operating condition of the power system; 2) the fault type and location; 3) the fault clearing time.

Stability can be evaluated by performing a time domain simulation of the power system under examination using a proper stability analysis software or alternatively, by using the so-called "direct methods" which avoid the integration of the system dynamic equations and provide stability indices. The simulated method can suit for different power system nonlinear models and the results are quite reliable, but it needs the repetitive transient stability runs to different original system conditions in order to obtain transient stability limits, and the calculating speed is very slow. Direct methods are analytical techniques based on the concepts of transient energy functions (TEF) developed in the last decades. Although these techniques are limited to first swing analysis, they are very interesting particularly for what concerns on-line analysis and dynamic security assessment.

The direct analysis method of the transient stability power limits is based on deriving analytical sensitivities of the energy $\Delta V$ to parameter changes and truncating the series expansion terms to the first order. Its calculation speed is faster than the simulated method, but stability limits obtained by using this method are very accurate only for cases where the parameter changes are not large or when the system is moderately stressed. However, when the changes in the energy margin to parameters are with higher nonlinear, the limits obtained by using this linearized technique are not very accurate. Although the second order series expansion terms of the energy margin sensitivity to parameter changes can be considered to overcome these nonlinear problems, it is difficult to obtain analytical expressions of second order sensitivities of the energy margin with respect to system parameter changes. On the other hand, because some assumptions have been used in order to reduce the calculating procedures of the analytical sensitivity of the energy margin, the accuracy of the transient stability limits is also affected.

In this paper, a novel method of calculating transient stability power limits is proposed using a self-organizing mapping $[8,9]$. Two methods are used to estimate the power security limits [10]. Lyapunov's direct method is used as a fast tool to obtain the training set of the neural network. The transient stability power limits of the generator are estimated at different fault clearing time and load levels.

\section{Transient Security Power Limits}

\section{A. System Model}

For a multimachine power system, the generator's equations of motion with respect to the centre of angle (COA) are generally denoted by:

$$
\begin{gathered}
M_{i} \frac{d \varpi_{i}}{d t}=\left(P_{m i}-P_{e i}\right)-\frac{M_{i}}{M_{T}} P_{a} \\
\frac{d \theta_{i}}{d t}=\sigma_{i} \quad(i=1, \cdots, n)
\end{gathered}
$$

where: $M_{T}$ is the total inertia, $M_{i}$ is the $i^{\text {th }}$ machine inertia, $P_{m i}$ is the mechanical power input and $P_{e i}$ the electrical power output of machine $i$.

\section{B. Transient Energy Function}

The training set of the neural network is obtained by synthetical method of fast direct transient stability analysis, which compositely uses the advantages of the three methods (EEAC, PEBS and RUEP) and is fault 
tolerance and with higher accuracy and reliability. The detailed mathematical model and calculating procedures of the synthetical direct method is given in appendix.

The expression of the individual transient energy function of machine $i$ is defined as:

$$
\dot{V}_{i}=\left(M_{i} \dot{\varpi}_{i}-P_{m i}+P_{e i}+\frac{M_{i}}{M_{T}} P_{a}\right) \dot{\theta}_{i}
$$

Since the determination of the critical energy function is time consuming due to the calculation of the unstable equilibrium points, a variable correlated to this energy is defined for a second order model [11] and can be expressed as:

$$
V_{c}=\sum_{i=1}^{n-1} \sum_{j=i+1}^{n} C_{i j}+\sum_{j=i+1}^{n} G_{i i} E_{q i}^{\prime 2}-\sum_{i=1}^{n} P_{m i}
$$

The calculation of this variable was generalised for a detailed mulimachine model including regulation systems.

\section{Estimation of Transient Stability Power Limits}

An inherent advantage of the direct method is the availability of a qualitative measure of the degree of stability (or instability) in terms of the transient energy margin $(\Delta V)$. This was considered as an important step toward reducing the number of transient stability runs needed to obtain transient limits to make changes to improve the system transient behaviour, when subjected to large disturbances. The quantitative answers for stability limits can be obtained by using the sensitivity of $\Delta V$ to changes in power system parameters from a base case operating condition. Since the value of the energy margin gives a direct indication of stability assessment, the sensitivity of the energy margin to specific changes in system conditions would give a direct estimate of the stability limits of these conditions.

In this paper, a new architecture of a combined neural network is proposed for fast estimation of transient stability power limits. The unsupervised learning system is used as a mechanism for fast screening of system disturbances. Input patterns are clustered according to similarities discovered among the input features. The range of label attributes (CCT) in each cluster indicates which associative actions should be taken in each instance. At the second stage, a supervised learning paradigm is performed to establish the nonlinear mapping relation among the transient energy margin and the generator power at different fault clearing time and load levels of the system. The established nonlinear mapping relation can be expressed by a function as:

$$
P=F\left(\Delta V, T_{c l}\right)
$$

where: $\Delta V=V_{c r}-V_{c l}$ is the transient energy margin,

with: $\quad V_{c r}$ : critical transient energy

$V_{c l}:$ transient energy at the time of fault clearing

$T_{c l}:$ fault clearing time

$P$ : active power of the studied generator
If the nonlinear mapping of the equation (4) is obtained, then we let $\Delta V=0$ (critical state), the transient stability power limits of the studied generator at fault clearing time $T_{c l}$ and a load

scenario can be found as a solution of the following equation:

$$
P_{\max }=F\left(0, T_{c l}\right)
$$

\section{Self-Organizing Mapping}

\section{A. Neural Network Architecture}

The conceptual diagram of the proposed algorithm is given in Figure1. The unsupervised neural network is the Growing Hierarchical Self-Organizing Map (GHSOM) and the supervised learning is the back error propagation with the Delta Rule (DR) algorithm.

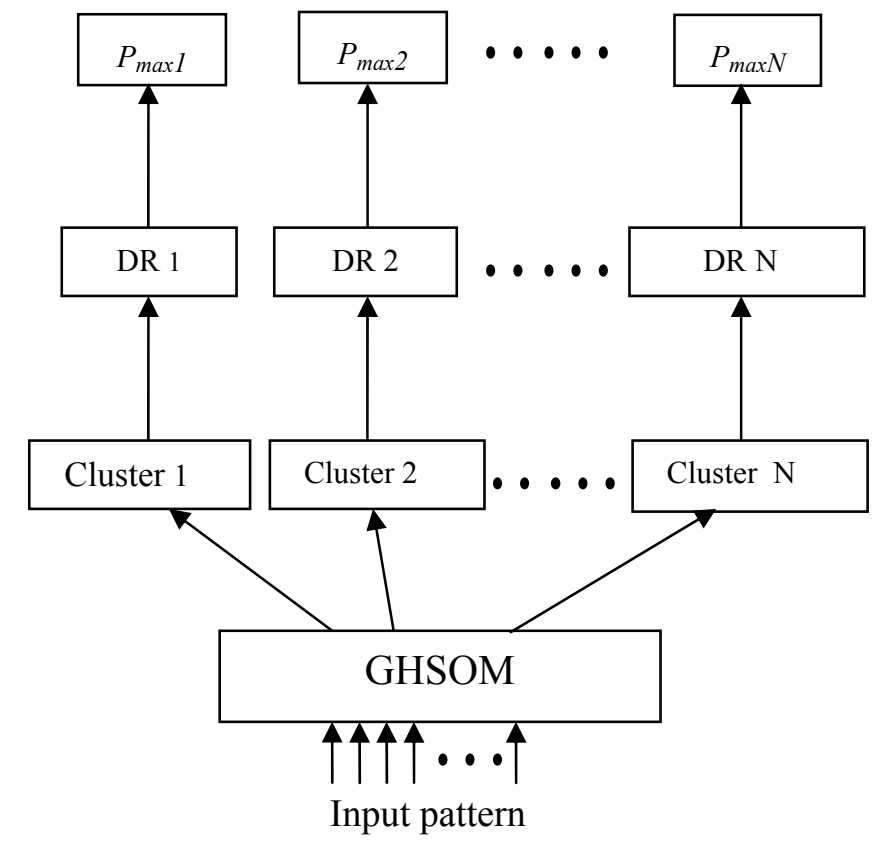

Figure 1. Proposed neural-net architecture

\section{B. The Growing Hierarchical Self-Organizing Map}

The choice of a clustering technique arises from the lack of a priori information of the problem. Unfortunately, SOM's require a previous knowledge of the problem to decide the shape of its grid. In order to overcome this limit, the GHSOM has been proposed [8]. The GHSOM has a hierarchical structure of multiple layers, where each layer consists of several independent growing SOMs.

A graphical representation of a GHSOM is given in Fig. 2 . The map in layer 1 consists of $3 \times 2$ units and provides a rather rough organization of the main clusters in the input data. The six independent maps in the second layer offer a more detailed view of the data. The input data for one map is the subset which has been mapped onto the corresponding unit in the upper layer. For the control of the growth process, two different strategies can be used, using 
either the mean quantization error (mqe) of a unit or the absolute value namely, the quantization error $(q e)$ of a unit.

The mqe of a unit $i$ is calculated according to (6) as the mean Euclidean distance between its model vector $m_{i}$ and the $n_{C}$ input vectors $x_{j}$ that are the set of input vectors $C_{i}$ mapped onto this unit $i$.

$$
m q \varepsilon=\frac{1}{n_{C}} \sum_{x_{j} \in C_{i}}\left|m_{i}-x_{j} \| \quad n_{C}=\right| C_{i} \mid, C_{i} \neq \phi
$$

The starting point for the GHSOM training process is the calculation of an $m q e_{0}$ of the unit forming the layer 0 map as provided in (4). With $n_{I}$ we refer to the number of all input vectors $x$ of the input data set $I$ and $m_{0}$ denotes the mean of the input data:

$$
m q e_{i}=\frac{1}{n_{I}} \sum_{x_{i} \in I}\left\|m_{0}-x_{i}\right\| \quad n_{I}=|I|
$$

The minimum quality of data representation of each unit will be specified as a fraction, indicated by a parameter $\tau$, of $m q e_{0}$ to satisfy the global termination criterion:

$$
m q_{i}<\tau . m q e_{0}
$$

For all units not satisfying this condition, a more detailed data representation is required, leading to the addition of further units to provide more map space for data representation.

The growth process of a growing SOM can be described as follows. Let $C_{i}$ be the subset of vectors $x_{j}$ of the input data that is mapped onto unit $i$, and $m_{i}$ the model vector of unit $i$. Then the error unit $e$ is determined as the unit with the mqe as follows:

$$
e=\arg \max _{i}\left(\sum_{x_{j} \in C_{i}}\left\|m_{i}-x_{j}\right\|\right) \quad n_{C}=\left|C_{i}\right|, C_{i} \neq \phi
$$

Following the selection of $e$, its most dissimilar neighbor $d$ is determined as follows, where $N_{e}$ is the set of neighboring units of $e$.

$$
d=\arg \max _{i}\left(\left\|m_{i}-x_{j}\right\|\right) \quad m_{i} \in N_{e}
$$

\section{Input and Output Features Selection}

Training patterns are obtained from simulations in which different pre-fault conditions and network topologies (single transmission line outages) are represented.

In the case of a geographical restricted surface power system, the variation in depth of the load plan is the most used to represent the homogeneous variation of the load demand. It can be obtained by multiplying all the active and reactive buses loads by a random number of uniform distribution varying between 0.8 and 1.2. However, in the case of a large power system with heterogeneous load demand variation in time, the variation in surface of the load is used by varying the loads independently.

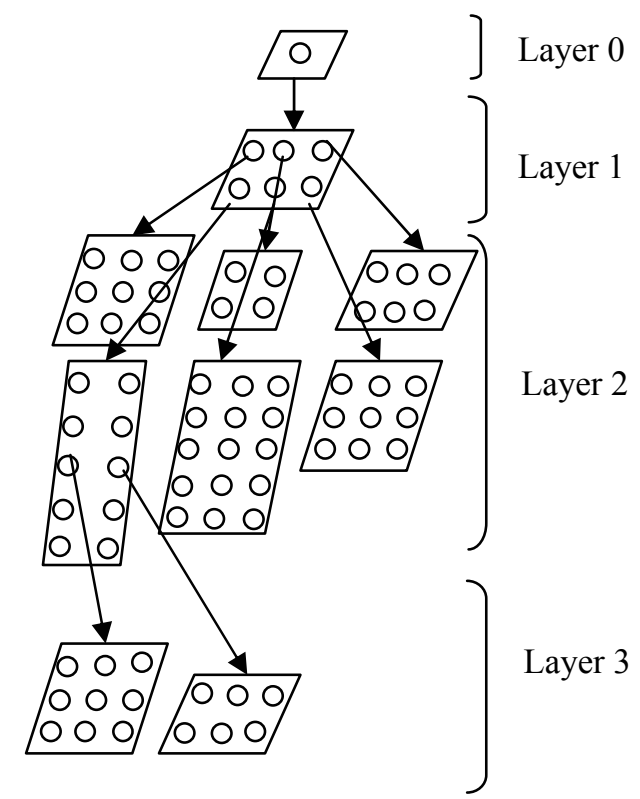

Figure 2. The GHSOM algorithm

The first step in the application of the proposed approach is to define a set of features in which input data will be represented. In this study, the input features of the unsupervised neural network are defined in terms of power system variables and they include:

1- The machines individual transient energy functions;

2- The variable correlated to the critical energy.

All patterns are labelled with the corresponding critical clearing time parameters.

The selected input features of the supervised neural network are the following variations:

1- Transient energy margin $\Delta V$

2- Fault clearing time $T_{c l}$

The output of the neural network is the active power of the studied generator in initial system operating conditions.

In obtaining the training set of the network, the following assumptions are made:

- The mode of disturbance does not change when system operating conditions are changed

- In each load level of the system, the shifts of generation at the studied machine is countered by an equal and opposite shift at the reference machine.

\section{Simulation Results}

The proposed approach is tested on a 7 machine CIGRE test system [12] shown in Figure 3. The fault case is bus 6 three phase short circuit, and the fault is cleaned by tripping the line 6-8. The fault clearing policy is to restore pre-fault system topology.

In all experiments, critical clearing time is found to be in the range between $0.19 \mathrm{~s}$ and $0.52 \mathrm{~s}$.

The GHSOM has 8 inputs and the corresponding training 
set is composed of the true post-fault state vectors (the base case and 12 single outage contingencies).

Based on the unit representing the mean of all data at layer 0 , the GHSOM training algorithm starts with a $2 \times 2 \mathrm{SOM}$ at the first layer. The training process for this map continues with the additional units being added until the quantization error drops below a percentage $\tau_{2}=0.005$ of the overall quantization error of the unit at layer 0 , resulting in $3 \times 2$ units representing six major clusters depicted in Figure 4.

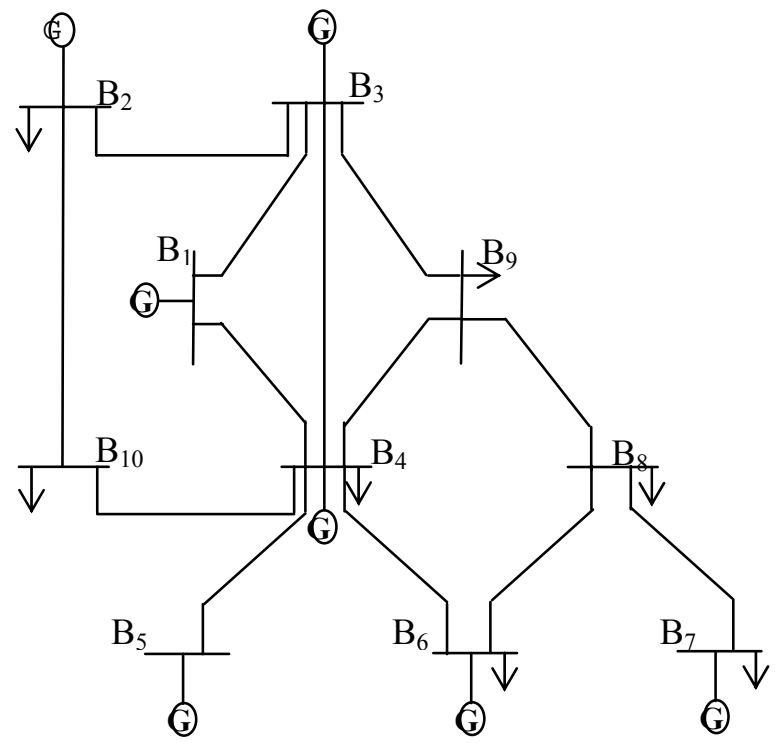

Figure 3. CIGRE Test System

Further, the notation $(i / j)$ is used to refer to the unit located in column $i$ and row $j$ of the map.

The main difference between these clusters lies in the range of the values reached by the critical clearing time. For example, unit (2/3) classifies the cases characterized by high values of the critical clearing time.

Further maps are automatically trained to represent the various state vectors, resulting in six individual maps on the second layer, each representing the data of the respective higher layer unit in more detail as $Q E_{m}$ reaches $\tau_{I}=0.08$ of the corresponding $q e$ 's unit in the layer 1 . In particular, we find a $(3 \times 2)$ map representing the patterns of unit $(1 / 2)$ of the first-layer map.

\begin{tabular}{|c|c|}
\hline $\begin{array}{l}0.19-0.27 \\
\underline{3 \times 1 ~ S O M}\end{array}$ & $\begin{array}{l}0.37-0.45 \\
\quad \underline{3 \times 2 \mathrm{SOM}}\end{array}$ \\
\hline $\begin{array}{l}0.26-0.33 \\
\underline{3 \times 2} \text { SOM }\end{array}$ & $\begin{array}{r}0.44-0.49 \\
3 \times 3 \text { SOM }\end{array}$ \\
\hline $\begin{array}{l}0.33-0.38 \\
\underline{2 \times 3 \mathrm{SOM}}\end{array}$ & $\begin{array}{c}0.48-0.52 \\
3 \times 2 \text { SOM }\end{array}$ \\
\hline
\end{tabular}

Figure 4. Layer 1 of the GHSOM

The studied generator is the critical machine (G6), which is severely disturbed and can be easily identified by the synthetical direct method. The severely disturbed generators also provide a greater effect on the energy margin.

The supervised neural network used in the case of unit $(1 / 2)$ consists of an input layer with 2 units, a two hidden layers with 6 units and 2 units respectively and an output layer with 1 unit. The number of the hidden layer units is not to be determined a priori, but adapts automatically to the learnt data.

In order to generate the training patterns, we varied the load levels of the system and the fault clearing time in range $0.05-0.25 \mathrm{~s}(0.05 \mathrm{~s}$ per step). The transient stability power limits at different fault clearing time are then obtained. In the testing mode, a group of new input features, not presented during the training session, is fed to the neural network with the transient energy margin $\Delta V=0$ (with respect to critical stable or unstable). The results for three Load Scenario (LS) are presented in Table1.

Table 1. Comparison of Actual and estimated Power Limits of machine 6

\begin{tabular}{|c|c|c|c|c|c|c|}
\hline \multirow{2}{*}{$\begin{array}{c}\text { Fault } \\
\text { Clearing }\end{array}$} & \multicolumn{5}{|c|}{ Power Limits (G6) } \\
\cline { 2 - 7 } Time $T_{c l}$ & \multicolumn{2}{|c|}{ LS 1 } & \multicolumn{2}{c|}{ LS 2 } & \multicolumn{2}{c|}{ LS 3 } \\
\cline { 2 - 7 }$(\mathrm{s})$ & ANN & SBS & ANN & SBS & ANN & SBS \\
\hline 0.05 & 1.87 & 1.82 & 1.88 & 1.84 & 1.88 & 1.86 \\
0.06 & 1.84 & 1.79 & 1.85 & 1.82 & 1.85 & 1.83 \\
0.07 & 1.80 & 1.76 & 1.82 & 1.79 & 1.82 & 1.81 \\
0.08 & 1.77 & 1.73 & 1.78 & 1.76 & 1.79 & 1.78 \\
0.09 & 1.74 & 1.70 & 1.75 & 1.73 & 1.76 & 1.75 \\
0.10 & 1.70 & 1.67 & 1.72 & 1.70 & 1.73 & 1.72 \\
0.11 & 1.66 & 1.64 & 1.68 & 1.66 & 1.70 & 1.69 \\
0.12 & 1.63 & 1.60 & 1.65 & 1.63 & 1.66 & 1.65 \\
0.13 & 1.59 & 1.57 & 1.61 & 1.58 & 1.63 & 1.62 \\
0.14 & 1.55 & 1.53 & 1.57 & 1.56 & 1.60 & 1.59 \\
0.15 & 1.51 & 1.50 & 1.54 & 1.53 & 1.56 & 1.55 \\
0.16 & 1.47 & 1.47 & 1.50 & 1.49 & 1.53 & 1.52 \\
0.17 & 1.44 & 1.43 & 1.46 & 1.46 & 1.50 & 1.49 \\
0.18 & 1.40 & 1.38 & 1.43 & 1.43 & 1.45 & 1.45 \\
0.19 & 1.36 & 1.36 & 1.39 & 1.39 & 1.42 & 1.42 \\
0.20 & 1.32 & 1.33 & 1.36 & 1.36 & 1.38 & 1.39 \\
0.21 & 1.29 & 1.30 & 1.32 & 1.33 & 1.35 & 1.36 \\
0.22 & 1.25 & 1.27 & 1.29 & 1.30 & 1.31 & 1.33 \\
0.23 & 1.22 & 1.24 & 1.26 & 1.27 & 1.28 & 1.30 \\
0.24 & 1.18 & 1.21 & 1.22 & 1.24 & 1.25 & 1.27 \\
0.25 & 1.15 & 1.18 & 1.19 & 1.21 & 1.21 & 1.24 \\
\hline
\end{tabular}

From Table 1, we can see that the estimated transient stability power limits agree with the results calculated by the time domain simulated method SBS (Step By Step).

\section{Conclusion}

In this paper, a new method for estimating transient stability power limits has been proposed. The main contribution is that the proposed method avoids the necessity of finding the analytical sensitivity of the transient energy margin to parameter changes. Another important aspect is that the proposed method can fast estimate transient security power limits at different fault clearing time and load levels of the system. Using unsupervised and supervised learning algorithms helps greatly to handle large bodies of data. With the growing hierarchical self organizing map, the overall training time is largely reduced since only the necessary number of units is developed to organise the state vectors and its architecture allows the user to understand and analyze large amounts of data in an explorative way. A 
supervised learning paradigm then uses the clustered data to synthesize accurately the transient stability power limit of the critical machine. The validity of the proposed method has been tested on a 7 machine-CIGRE test system and by comparison with the time domain simulations, close agreements of neural network predictions and actual transient stability power limits have been demonstrated. Our further work is to consider its application to large scale power systems

\section{References}

[1] M. Pavella , P.G. Murthy, Transient stability of power Systems - Theory and practice, John Willey \& Sons, Chichister, England, 1994.

[2] A.A. Fouad and V. Vittal., Power system transient stability analysis using the transient energy function method, Prentice Hall, Englewood, New Jersey, USA, 1992.

[3] M. Balu, et al., "On-line power system security analysis", Proceeding of the IEEE, Vol. 80, n², pp. 260-280, 1992.

[4] F.A. Rahimi, et al., 'Evaluation of the transient energy function method for on-line dynamic security assessment', IEEE trans. on Power Systems, Vol. 8, n², pp. 497-507, May 1993.

[5] V. Vittal, et al., " Derivation of stability limits using analytical sensitivity of the transient energy margin", IEEE trans. on Power Systems, Vol. 4, n4, pp. 1363-1369, 1989.

[6] D.Z. Fang, et al., " Transient stability limit conditions analysis using a corrected transient energy function approach", IEEE trans. on Power Systems, Vol. 15, n², pp. 804-810, May 2000.

[7] I. Kamwa, et al., " Time varying contingency screening for dynamic security assessment using intelligent-systems techniques", IEEE trans. on Power Systems, Vol. 16, n³, pp. 526-810, August 2001.

[8] T. Kohonen, Fast evolutionary learning with batch-type selforganizing maps, Neural Process Lett, 1999.

[9] S. Kask, et al., Bibliography of self-organizing map (SOM) papers, Neural Comput-Surveys, Vol. 1, No. 3 \& 4, pp. 1176, 1998.

[10] M. Boudour and A. Hellal, ’ Self-Organizing feature maps for power system dynamic security assessment using synchronizing and damping torques technique", Proceedings of the $29^{\text {th }}$ Annual Conference of the IEEE Industrial Electronics Society, Roanoke, Virginia, USA, pp. 752-758, November 2003.

[11] D.Z. Fang, et al., "' Transient stability limit conditions analysis using a corrected transient energy function approach", IEEE trans On Power Systems, Vol.15, n², pp. 804-810, May 2000.

\section{Appendices}

\section{Appendix A: Multimachine Model with Regulation Systems}

$$
\begin{aligned}
& \frac{d E_{d i}^{\prime}}{d t}=\frac{1}{T_{d o i}^{\prime}}\left(-\frac{X_{q i}}{X_{d i}^{\prime}} E_{d i}^{\prime}-\frac{\left(X_{d i}^{\prime}-X_{q i}\right)}{X_{d i}^{\prime}} V_{d i}\right) \\
& \frac{d E_{q i}^{\prime}}{d t}=\frac{1}{T_{d o i}^{\prime}}\left(-\frac{X_{d i}}{X_{d i}^{\prime}} E_{q i}^{\prime}+E_{f d i}-\frac{\left(X_{d i}^{\prime}-X_{d i}\right)}{X_{d i}^{\prime}} V_{q i}\right) \\
& \frac{d E_{f d i}}{d t}=\frac{1}{T_{A i}}\left(-E_{f d i}+E_{f d 0 i}+K_{A i}\left(V_{r e f i}-\sqrt{V_{d i}^{2}+V_{q i}^{2}}\right)\right)
\end{aligned}
$$

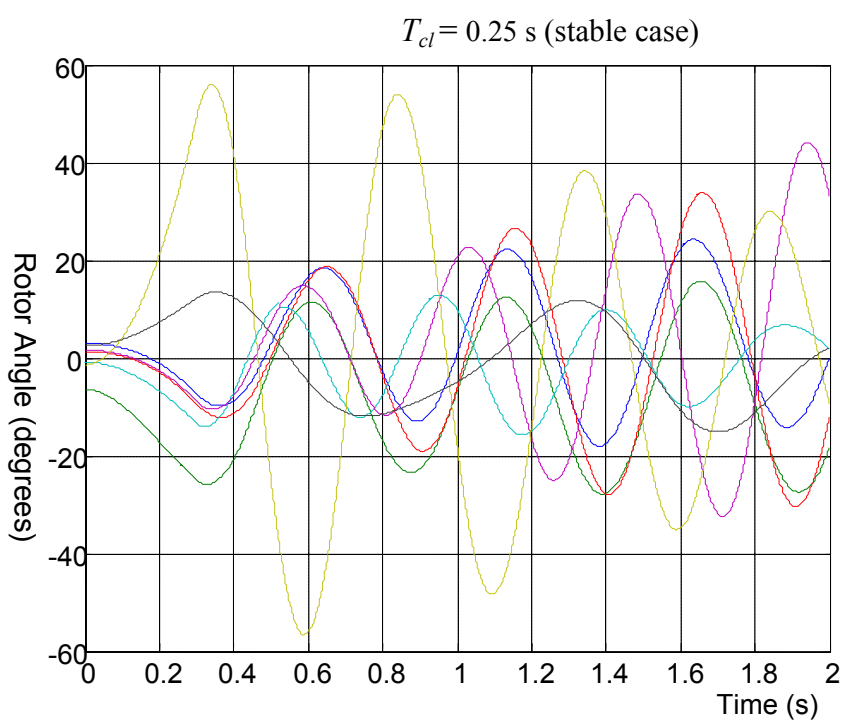

Figure A.1 7 machines System Rotor Angles

$$
\begin{aligned}
& \frac{d E_{d i}^{\prime}}{d t}=\frac{1}{T_{q o i}^{\prime}}\left(E_{d i}^{\prime}+\left(X_{d i}^{\prime}-X_{q i}\right) I_{q i}\right) \\
& \frac{d E_{q i}^{\prime}}{d t}=\frac{1}{T_{d o i}^{\prime}}\left(-E_{q i}^{\prime}+E_{f d i}-\left(X_{d i}^{\prime}-X_{d i}\right) I_{d i}\right) \\
& \frac{d \omega_{i}}{d t}=\frac{1}{M_{i}}\left(P_{m i}-P_{e i}\right) \\
& \frac{d \delta_{i}}{d t}=\omega_{i} \\
& \frac{d E_{f d i}}{d t}=\frac{1}{T_{A i}}\left(-E_{f d i}^{\prime}+E_{f d}+K_{A i}\left(V_{r e f i}-V_{i}\right)\right) \\
& \frac{d P_{m i}}{d t}=\frac{1}{T_{v i}}\left(-P_{m i}+P_{m r e f i}-K_{v i}\left(\omega_{i}+\sigma_{i} \frac{d \omega_{i}}{d t}\right)\right)
\end{aligned}
$$

\section{Appendix B: Transient Energy Function}

The critical energy function can be expressed as:

$$
\begin{aligned}
V_{c r}=\sum_{i=1}^{n} & V_{i}\left(t_{0}, t_{e}\right)=\sum_{j=1}^{n}\left(G_{i i} E_{q i}^{\prime 2}-P_{m i}\right)\left(\theta_{i}^{s}-\theta_{i}^{c}\right) \\
& +\sum_{i=1}^{n-1} \sum_{j=i+1}^{n} C_{i j}\left(\cos \theta_{i j}^{s}-\cos \theta_{i j}^{c}\right) \\
& +\sum_{i=1}^{n-1} \sum_{j=i+1}^{n} D_{i j} \int_{\theta_{i}^{s}+\theta_{j}^{s}}^{\theta_{j}^{c} \cos \theta_{j}^{c}} \theta_{i j} d\left(\theta_{i}+\theta_{j}\right)
\end{aligned}
$$

where:

$\left(\theta_{i}^{s}, 0\right)$ : post-fault stable equilibrium point $\left(\theta_{i}^{c}, 0\right)$ : Controlling Unstable Equilibrium Point (CUEP) The separation of the system generators into a stable cluster and an unstable cluster permits to use in a corrected kinetic energy $V_{\text {KEcorr }}$ which does not include the part of kinetic energy responsible for intermachine oscillations. An index normalised with respect to $V_{K E c o r r}$ $\left(\Delta V_{n}=\Delta V / V_{K E c o r r}\right)$ is particularly useful when comparing severity of each contingency. 